\title{
On the free boundary min-max geodesics
}

\author{
Xin Zhou*
}

July 30, 2018

\begin{abstract}
Given a Riemannian manifold and a closed submanifold, we find a geodesic segment with free boundary on the given submanifold. This is a corollary of the min-max theory which we develop in this article for the free boundary variational problem. In particular, we develop a modified Birkhoff curve shortening process to achieve a strong "Colding-Minicozzi" type min-max approximation result.
\end{abstract}

\section{Introduction}

Let $\left(M^{n}, g\right)$ be a complete and homogeneously regular Riemannian manifold, and $N^{m}$ a closed submanifold. We consider the problem of finding a geodesic in $M$ with end points on $N$, which meets $N$ orthogonally. This is a variational problem with free boundary conditions. We are interested in the case $\pi_{1}(M, N)=0$, where every curve with end points on $N$ can be shrunk to a point. Hence direct variational method fails in this situation, and we explore the min-max methods. Similar idea was first given by Birkhoff in the 1910s to find closed geodesics on the 2-sphere [B, V.7] (See [CM11, §5][Cr88, §2] for more details). In brief, given a sweepout, i.e. 1-parameter family of closed curves which cover the 2-sphere, Birkhoff developed a curve shortening process to make each slice of the sweep-out as tight as possible, and then obtained a closed geodesic as the limit of slices with maximal length. Since then, more results, such as the existence of multiple geodesics or even infinitely many geodesics, have been studied extensively (c.f. [Kl82, Gr]). Recently, a strong version was given by Colding and Minicozzi CMM08, where they found good approximating sweep-outs, such that "every curve in the sweepouts with length close to the longest must be close to a closed geodesic" (see [LW] for a proof using harmonic map flow).

In this paper, we develop a version of min-max methods for free boundary geodesics with a strong approximation result in the sense of Colding-Minicozzi, i.e. "every curve in the tightened sweepouts with length close to the longest must be close to a free boundary geodesic". The existence of a nontrivial free boundary geodesic is then a direct corollary. Such strong property is shared by many min-max constructions of critical points for variational problems, c.f. [AF65, §12.5][P81, §4.3] CD03, Proposition 3.1] CMM08, Theorem 1.9][CMR08, Theorem

\footnotetext{
${ }^{*}$ The author is partially supported by NSF grant DMS-1406337.
} 
1.14], and is very useful in other geometric problems, e.g. the proof of finite time extinction of 3-dimensional Ricci flow [CMR08].

The existence of geodesic with free boundary was discussed in various special cases before. Weinstein produced free boundary geodesic in a standard ball with Finsler metrics [W, §4], by embedding the ball to a sphere. Nabutovsky and Rotman [NR] studied the multiple solutions of geodesic loops where the constrained submanifold $N$ is a point. Compared to them, we extend the existence result to the full generality - the total space $M$ and the constraint $N$ can be any manifold and submanifold.

Besides the case of geodesics, min-max methods have been studied widely in high dimensions [P81, Jo89, F00, CD03, CMR08, MN12, L14]. Among them, the free boundary conditions were studied by Fraser [F00] in the case of harmonic disks, and by Jost [Jo89] and Li [L14] in the case of embedded minimal disks.

Let us introduce the notations and state the main results here. By the Nash embedding theorem, we can assume that $(M, g)$ is isometrically embedded in some Euclidean space $\mathbb{R}^{N}$. Denote $d s_{0}^{2}$ by the Euclidean metric. By scaling, we can assume that

(M1) $\sup _{M}\left\|A_{M}\right\| \leq \frac{1}{16}, \sup _{N}\left\|A_{N}\right\| \leq \frac{1}{16}$, where $A_{M}$ and $A_{N}$ are the second fundamental forms of the embedding $M \subset \mathbb{R}^{N}$ and $N \subset \mathbb{R}^{N}$ respectively;

(M2) The injective radius of $M$ is at least 8 , and the curvature of $M$ is at most $\frac{1}{64}$;

$(\mathrm{M} 2)^{\prime}$ The injective radius of $N$ is at least 4 , and the focal radius of $N$ is at least 4;

(M3) For any $x, y \in M$, with $|x-y|_{d s_{0}^{2}} \leq 8$, $\operatorname{dist}_{M}(x, y) \leq 2|x-y|$;

$(\mathrm{M} 3)^{\prime}$ For any $x, y \in N$, with $|x-y|_{d s_{0}^{2}} \leq 8$, $\operatorname{dist}_{N}(x, y) \leq 2|x-y|$.

Remark 1.1. If $M$ is compact, then the above constraints can be easily achieved by scaling. When $M$ is noncompact, we can assume that the conditions hold in a large convex domain containing $N$.

Let $I=[0,1]$ be the unit interval, and we will work in the Sobolev space $W^{1,2}(I, M)$, where the $W^{1,2}$-norm of a map $f: I \rightarrow M \subset \mathbb{R}^{N}$ is given by

$$
\|f\|_{W^{1,2}}^{2}=\int_{[0,1]}|f(x)|^{2}+\left|f^{\prime}(x)\right|^{2} d x .
$$

The energy of $f$ is defined by

$$
E(f)=\int_{[0,1]}\left|f^{\prime}(x)\right|^{2} d x .
$$

Now we can define the total variational space as follows:

Definition 1.2. Let $\Omega$ be the space of continuous mappings $\sigma:[0,1] \times I \rightarrow M$, such that using coordinates $(t, x) \in[0,1] \times I$,

- $\sigma(t, \cdot) \in W^{1,2}(I, M)$, and $\sigma(t, 0), \sigma(t, 1) \in N$, for all $t \in[0,1]$

- $t \rightarrow \sigma(t, \cdot)$ is continuous as a map from $[0,1]$ to $W^{1,2}(I, M)$; 
- $\sigma(0, \cdot), \sigma(1, \cdot)$ are constant maps.

Each $\sigma$ is called a sweep-out.

Remark 1.3. The notion of sweep-out comes from the special case when $(M, \partial M)$ is diffeomorphic to the unit disk $(D, \partial D)$, then we can choose $\sigma(t)$ to sweep out the whole disk.

Given $\sigma_{0} \in \Omega$, we let $\left[\sigma_{0}\right]$ to be the set of all $\sigma \in \Omega$ which is homotopic to $\sigma_{0}$ in $\Omega$. Then we can define the width associated to $\sigma_{0}$ as:

$$
W=W\left(\left[\sigma_{0}\right]\right)=\inf _{\sigma \in\left[\sigma_{0}\right]} \max _{t \in[0,1]} E(\sigma(t)) .
$$

The next result says that the width is always achieved by a geodesic of $M$ with free boundary on $N$.

Theorem 1.4. Given $\sigma_{0} \in \Omega$, with $W\left(\left[\sigma_{0}\right]\right)>0$, there exists a nontrivial geodesic $\gamma: I \rightarrow M$ with free boundary on $N$, i.e. $\gamma(0), \gamma(1) \in N, \gamma^{\prime}(0), \gamma^{\prime}(1) \perp N$, and $E(\gamma)=W_{\left[\sigma_{0}\right]}$.

Remark 1.5. When $N$ bounds a non-contractable disk in $M$, we can find $\sigma_{0}$ with $W\left(\left[\sigma_{0}\right]\right)>0$ (see the discussion in the beginning of 4 (4).

Theorem 1.4 is a direct corollary of the following stronger theorem, which says that "almost maximal implies almost critical".

Theorem 1.6. Given $\sigma_{0} \in \Omega$, with $W\left(\left[\sigma_{0}\right]\right)=W_{0}>0$, then there exists a sequence of sweep-outs $\left\{\gamma_{j}\right\}_{j \in \mathbb{N}} \subset\left[\sigma_{0}\right]$, with

$$
\lim _{j \rightarrow \infty} \max _{t \in[0,1]} E\left(\gamma_{j}(t)\right)=W_{0}
$$

such that, for any $\epsilon>0$, there exists $a \delta>0$, and if $j>\frac{1}{\delta}$, and if for some $t_{0} \in[0,1]$

$$
E\left(\gamma_{j}\left(t_{0}\right)\right)>W_{0}-\delta
$$

then $\operatorname{dist}\left(\gamma_{j}\left(t_{0}\right), G\right)<\epsilon$, where $G$ is the space of immersed geodesics with free boundary on $N$.

Remark 1.7. $\gamma_{j}(t)$ will be piecewise geodesic by our construction, so $E\left(\gamma_{j}\left(t_{0}\right)\right)=\operatorname{Length}^{2}\left(\gamma_{j}\left(t_{0}\right)\right)$, hence (1.2) says that the length of $\gamma_{j}\left(t_{0}\right)$ is almost maximal among the sweep-out $\gamma_{j}(t)$.

The idea is to adapt the Birkhoff's curve shortening process (BCSP) to manifold with a constraint submanifold. Briefly, given a closed curve $\gamma$ with $2 L$ evenly spaced break points $\left\{x_{0}, x_{1}, \cdots, x_{2 L}=x_{0}\right\} \subset S^{1}$, the BCSP first replaces each piece $\left.\gamma\right|_{\left[x_{2 i}, x_{2 i+2}\right]}$ on even intervals by a geodesic segment connecting $\gamma\left(x_{2 i}\right)$ with $\gamma\left(x_{2 i+2}\right)$, and then repeats the geodesic replacement process on odd intervals $\left[x_{2 i-1}, x_{2 i+1}\right]$. In our case, given a curve $\gamma:[0,1] \rightarrow M$, $\gamma(0), \gamma(1) \in N$, with $2 L+1$ evenly spaced break points $\left\{x_{0}=0, x_{1}, \cdots, x_{2 L}=1\right\} \subset[0,1]$, we will first replace the boundary piece $\left.\gamma\right|_{\left[0, x_{2}\right]}$ (and $\left.\gamma\right|_{\left[x_{2 L-2}, 1\right]}$ ) by geodesic segment $\tilde{\gamma}$ connecting $\gamma\left(x_{2}\right)$ (and $\gamma\left(x_{2 L-2}\right)$ ) to $\mathrm{N}$, and then do replacements on inner pieces $\left.\gamma\right|_{\left[x_{2 i}, x_{2 i+2}\right]}, i \neq 0, L-1$ and $\left.\gamma\right|_{\left[x_{2 i-1}, x_{2 i+1}\right]}$ as BCSP. Our modified BCSP satisfies properties analogous to BSCP (\$3). 
Among them, one key ingredient is to show that the $W^{1,2}$-norm difference $\left\|\left.\gamma\right|_{\left[0, x_{2}\right]}-\tilde{\gamma}\right\|_{1,2}$ is controlled by the length difference $\operatorname{Length}\left(\left.\gamma\right|_{\left[0, x_{2}\right]}\right)-\operatorname{Length}(\tilde{\gamma})(\operatorname{Lemma} 3.2)$. This is achieved by certain convexity estimates, which will be useful in other free boundary variational problems.

The paper is organized as follows. In 92 , we collect several preliminary results. In 93 , we introduce the modified curve shortening process and several key properties. In \$4, we apply the curve shortening process to sweep-outs and finish the proof.

Acknowledgement: This paper is based upon work supported by the National Science Foundation under Grant No. 0932078 000, while the author was visiting the Mathematical Science Research Institute in Berkeley, California, during the Fall semester of 2013. The author would like to than Rick Schoen, Toby Colding and Bill Minicozzi for discussions.

\section{Preliminary results}

Now we summarize several preliminary results in this section. The first fact is that the $W^{1,2}$-norm bounds imply the Hölder continuity, i.e. given $x, y \in I$, using the Cauchy-Schwartz inequality,

$$
|f(x)-f(y)|^{2} \leq\left(\int_{x}^{y}\left|f^{\prime}\right|\right)^{2} \leq|x-y| \int_{I}\left|f^{\prime}\right|^{2} .
$$

Thus $f$ is in $C^{\frac{1}{2}}$ if $f$ is in $W^{1,2}$, and the $C^{\frac{1}{2}}$-norm is bounded by the $W^{1,2}$-norm.

The second important result is the Wirtinger inequality and the modified Wirtinger inequality. Let us first state the Wirtinger inequality, which was used a lot in studying the existence of closed geodesics by Colding and Minicozzi [CM11, page 165]. Given $L>0$, let $f \in W^{1,2}([0, L], \mathbb{R})$, with $f(0)=f(L)=0$, then

$$
\int_{0}^{L}(f(x))^{2} d x \leq \frac{L^{2}}{\pi^{2}} \int_{0}^{L}\left|f^{\prime}(x)\right|^{2} d x .
$$

For the modified Wirtinger inequality, we want similar estimates, while only assuming that the function is zero at one of the boundary points of $[0, L]$. Precisely, we have

Lemma 2.1. Let $f \in W^{1,2}([0, L], \mathbb{R})$, with $f(0)=0$, then

$$
\int_{0}^{L}(f(x))^{2} d x \leq \frac{L^{2}}{2} \int_{0}^{L}\left|f^{\prime}(x)\right|^{2} d x .
$$

Proof. Using the fundamental theorem of calculus, $f(x)=\int_{0}^{x} f^{\prime}(s) d s$. By the CauchySchwartz inequality,

$$
|f(x)|^{2} \leq\left(\int_{0}^{x}\left|f^{\prime}(s)\right| d s\right)^{2} \leq x \int_{0}^{L}\left|f^{\prime}(s)\right|^{2} d s
$$

So

$$
\int_{0}^{L}|f(x)|^{2} d x \leq \int_{0}^{L} x\left(\int_{0}^{L}\left|f^{\prime}(s)\right|^{2} d s\right) d x=\frac{L^{2}}{2} \int_{0}^{L}\left|f^{\prime}(s)\right|^{2} d s .
$$


The last fact is as follows. It appears in [CM11, Lemma 5.2], and we need a version with better constants. It just comes out as a careful reproof of [CM11, Lemma 5.2], and we give the details here for completeness.

Lemma 2.2. If $x, y \in M$, then $\left|(x-y)^{\perp}\right| \leq \frac{1}{8}|x-y|^{2}$, where $(x-y)^{\perp}$ is the normal component of $(x-y)$ to $M$ at $y$ in $\mathbb{R}^{N}$. Similar inequality also holds when $x, y \in N$, and when we take $(x-y)^{\perp}$ to be the normal component of $(x-y)$ to $N$ at $y$ in $\mathbb{R}^{N}$.

Proof. If $|x-y| \geq 8$, then it is trivially true. Assume now that $|x-y| \leq 8$. Choose $\alpha:[0, l] \rightarrow$ $M$ as the minimizing unit speed geodesic from $y$ to $x$ in $M$ (which exists by (M2)), hence $l=\operatorname{dist}_{M}(x, y) \leq 2|x-y|$ by (M3). Let $V$ be the unit normal vector

$$
V=\frac{(x-y)^{\perp}}{\left|(x-y)^{\perp}\right|}
$$

so $\left\langle\alpha^{\prime}(0), V\right\rangle=0$. Hence

$$
\begin{aligned}
\left|(x-y)^{\perp}\right| & =\langle(x-y), V\rangle=\int_{0}^{l}\left\langle\alpha^{\prime}(s), V\right\rangle d s=\int_{0}^{l}\left\langle\alpha^{\prime}(0)+\int_{0}^{s} \alpha^{\prime \prime}(t) d t, V\right\rangle d s, \\
& \leq \int_{0}^{l} \int_{0}^{s}\left|\alpha^{\prime \prime}(t)\right| d t d s \leq \int_{0}^{l} \int_{0}^{s}\left|A_{M}(\alpha(t))\right|\left|\alpha^{\prime}(t)\right|^{2} d t d s, \\
& \leq \frac{1}{2} l^{2} \sup _{M}\left|A_{M}\right| \leq \frac{1}{8}|x-y|^{2} .
\end{aligned}
$$

The same proof works for $N$, as it satisfies the same properties as $M$ as embedded submanifolds of $\mathbb{R}^{N}$.

\section{Curve shortening process}

Take $L \in \mathbb{N}$ to be a large integer. Let $\Lambda$ be the space of piecewise linear maps 1 $\gamma: I \rightarrow M$ parametrized proportional to the arc-length with no more than $L-1$ break points, such that $\gamma(0), \gamma(1) \in N$, and each geodesic segment has length at most 1 , with Lipschitz bound $L$. Denote $G \subset \Lambda$ to be the set of immersed geodesics with free boundary lying on $N$. We will use the distance and topology on $\Lambda$ given by the $W^{1,2}$-norms on $W^{1,2}(I, M)$.

In this section, we will construct the modified Birkhoff curve shortening map $\Psi: \Lambda \rightarrow \Lambda$, so that the following properties are satisfied2:

(1) $\Psi(\gamma)$ depends on $\gamma$ continuously;

(2) $\Psi(\gamma)$ is homotopic to $\gamma$, and Length $(\Psi(\gamma)) \leq \operatorname{Length}(\gamma)$;

(3) There is a continuous function $\phi:[0, \infty) \rightarrow[0, \infty)$, with $\phi(0)=0$, such that,

$$
\operatorname{dist}^{2}(\gamma, \Psi(\gamma)) \leq \phi\left(\frac{\operatorname{Length}^{2}(\gamma)-\operatorname{Length}^{2}(\Psi(\gamma))}{\operatorname{Length}^{2}(\Psi(\gamma))}\right) ;
$$

\footnotetext{
${ }^{1}$ By linear map, we mean a (constant speed) geodesic.

${ }^{2}$ Similar map and properties appear in CM11 page 165] in the case of closed curves.
} 
(4) If $\Psi(\gamma)=\gamma$, then $\gamma \in G$, i.e. fixed points of $\Psi$ are immersed geodesics with free boundary lying on $N$;

(5) Given $\epsilon>0$, there exists $\delta>0$, such that if $\gamma \in \Lambda$, and $\operatorname{dist}(\gamma, G) \geq \epsilon$, then

$$
\operatorname{Length}(\Psi(\gamma)) \leq \operatorname{Length}(\gamma)-\delta
$$

3.1 Defining $\Psi$. Fix a partition of $I=[0,1]$ by choosing $2 L-1$ successive evenly spaced break points:

$$
x_{0}=0, x_{1}, x_{2}, \cdots, x_{2 L}=1 \in[0,1],
$$

such that $\left|x_{j+1}-x_{j}\right|=\frac{1}{2 L}$. Similarly to that in [CM11], $\Psi$ is defined by four steps:

Step 1. Replace $\gamma$ on the boundary even intervals $\left[0, x_{2}\right]$ and $\left[x_{2 L-2}, 0\right]$ by the minimizing geodesics from $\gamma\left(x_{2}\right)$ and $\gamma\left(x_{2 L-2}\right)$ to $N$ respectively 3 , and then replace $\gamma$ on each inner even interval $\left[x_{2 j}, x_{2 j+2}\right]$ by the linear map with the same endpoints to get a piecewise linear map $\gamma_{e}:[0,1] \rightarrow M$.

Step 2. Reparametrize $\gamma_{e}$ to get a constant speed curve $\tilde{\gamma}_{e}$, i.e. $\tilde{\gamma}_{e}$ is parametrized proportional to the arc length.

Step 3. Denote $\tilde{x}_{j}$ to be the image of $x_{j}$ under this reparametrization, i.e. $\gamma_{e}\left(x_{j}\right)=\tilde{\gamma}_{e}\left(\tilde{x}_{j}\right)$. Replace $\tilde{\gamma}_{e}$ on each odd interval $\left[\tilde{x}_{2 j-1}, \tilde{x}_{2 j+1}\right]$ by the linear map with the same endpoints to get a piecewise linear map $\gamma_{o}:[0,1] \rightarrow M$.

Step 4. Reparametrize $\gamma_{o}$ to get a constant speed curve $\tilde{\gamma}_{o}$, which is then $\Psi(\gamma)$.

In fact, each of the steps is energy non-increasing. The linear replacements obviously reduce the energy, as the linear maps (with both fixed endpoints, or with one endpoint fixed and the other free on $N$ ) minimize energy. The reparametrizations reduce the energy because of the Cauchy-Schwartz inequality, since for a map $\gamma:[0,1] \rightarrow M$,

$$
\operatorname{Length}^{2}(\gamma) \leq E(\gamma)
$$

where equality holds if and only if $\gamma$ has constant speed almost everywhere. Now we will prove these properties of $\Psi$ in the following.

3.2 Property (3) of $\Psi$. Using the triangle inequality, we only need to bound $\operatorname{dist}\left(\gamma, \gamma_{e}\right)$ and $\operatorname{dist}\left(\gamma_{e}, \tilde{\gamma}_{e}\right)$ by the the difference of their length square (as well as those in Step 3 and Step 4). In CMM08, CM11, Colding and Minicozzi showed that the $W^{1,2}$-distance of two curves $\sigma, \tilde{\sigma}$ can be bounded by the difference of their length square when $\tilde{\sigma}$ is a linear map with the same endpoints as $\sigma$, and they also showed analogous bound for the reparametrization as Step 2 and Step 4. Hence the bounds of $\operatorname{dist}\left(\tilde{\gamma}_{e}, \gamma_{o}\right)$ and $\operatorname{dist}\left(\gamma_{o}, \tilde{\gamma}_{o}\right)$ are almost the same as those in [CM11, §3.2], except that the parameter space is $[0,1]$ instead of $S^{1}$. The only estimate left in our case is to find similar bound of $\operatorname{dist}(\sigma, \tilde{\sigma})$ when $\sigma$ has one endpoint on $N$ and $\tilde{\sigma}$ is the minimizing geodesic from the other endpoint of $\sigma$ to $N$. Particularly,

\footnotetext{
${ }^{3}$ Such geodesics exist as $\gamma\left(x_{2}\right)$ and $\gamma\left(x_{2 L}-2\right)$ lie within the boundary cut locus by (M2)'.
} 
Lemma 3.1. (CM11, Lemma 5.1]) There exists $C$ so that if $I$ is an interval of length at most $\frac{1}{L}, \sigma_{1}: I \rightarrow M$ is a Lipschitz curve with $\left|\sigma_{1}^{\prime}\right| \leq L$, and $\sigma_{2}: I \rightarrow M$ is the minimizing geodesic with the same endpoints, then

$$
\operatorname{dist}^{2}\left(\sigma_{1}, \sigma_{2}\right) \leq C\left(E\left(\sigma_{1}\right)-E\left(\sigma_{2}\right)\right)
$$

We have the analog for the free boundary case.

Lemma 3.2. There exists $C^{\prime}$ so that if $I$ is an interval of length at most $\frac{1}{L}$, say $I=[0, l]$, $l \leq \frac{1}{L}, \sigma_{1}: I \rightarrow(M, N)$ is a Lipschitz curve with $\left|\sigma_{1}^{\prime}\right| \leq L$, with one endpoint lying on $N$, i.e. $\sigma_{1}(l) \in N$, and $\sigma_{2}: I \rightarrow(M, N)$ is the minimizing geodesic from $\sigma_{2}(0)$ to $N$, i.e. $\sigma_{2}(0)=\sigma_{1}(0), \sigma_{2}(l) \in N$ and $\sigma_{2}^{\prime}(l) \perp N$, then

$$
\operatorname{dist}^{2}\left(\sigma_{1}, \sigma_{2}\right) \leq C^{\prime}\left(E\left(\sigma_{1}\right)-E\left(\sigma_{2}\right)\right) \text {. }
$$

Proof. Integration by part and using that $\sigma_{1}$ and $\sigma_{2}$ are equal at 0 ,

$$
\begin{aligned}
\int_{I}\left|\sigma_{1}^{\prime}\right|^{2}-\int_{I}\left|\sigma_{2}^{\prime}\right|^{2} & -\int_{I}\left|\sigma_{1}^{\prime}-\sigma_{2}^{\prime}\right|^{2}=2 \int_{I}\left\langle\sigma_{2}^{\prime},\left(\sigma_{1}-\sigma_{2}\right)^{\prime}\right\rangle d s \\
& =\left.2\left\langle\sigma_{2}^{\prime},\left(\sigma_{1}-\sigma_{2}\right)\right\rangle\right|_{s=l}-2 \int_{I}\left\langle\left(\sigma_{1}-\sigma_{2}\right), \sigma_{2}^{\prime \prime}\right\rangle d s .
\end{aligned}
$$

For the first term in the last line, as $\sigma_{2}$ is a minimizing geodesic, with length less or equal than that of $\sigma_{1},\left|\sigma_{2}^{\prime}\right| \leq L$. Also by the fundamental theorem of calculus, the Cauchy-Schwartz inequality, and the fact that $\sigma_{1}(0)=\sigma_{2}(0)$,

$$
\begin{aligned}
\left|\left(\sigma_{1}-\sigma_{2}\right)(l)\right| & =\left|\int_{0}^{l}\left(\sigma_{1}-\sigma_{2}\right)^{\prime} d s\right| \leq \int_{0}^{l}\left|\left(\sigma_{1}-\sigma_{2}\right)^{\prime}\right| d s \\
& \leq l^{\frac{1}{2}}\left(\int_{0}^{l}\left|\left(\sigma_{1}-\sigma_{2}\right)^{\prime}\right|^{2} d s\right)^{\frac{1}{2}} .
\end{aligned}
$$

Since $\sigma_{2}^{\prime}(l)$ is normal to $N$, we can use Lemma 2.2 for $x=\sigma_{1}(l) \in N$ and $y=\sigma_{2}(l) \in N$, hence

$$
\begin{aligned}
\left|\left\langle\sigma_{2}^{\prime},\left(\sigma_{1}-\sigma_{2}\right)\right\rangle\right|_{s=l} \mid & \leq \frac{1}{8}\left|\sigma_{2}^{\prime}(l)\right| \cdot\left|\left(\sigma_{1}-\sigma_{2}\right)(l)\right|^{2} \leq \frac{1}{8} L \cdot l\left(\int_{0}^{l}\left|\left(\sigma_{1}-\sigma_{2}\right)^{\prime}\right|^{2} d s\right) \\
& \leq \frac{1}{8}\left(\int_{I}\left|\sigma_{1}^{\prime}-\sigma_{2}^{\prime}\right|^{2} d s\right) .
\end{aligned}
$$

For the second term in the last line of (3.3), we can use similar argument as in CMM08, CM11]. Using the geodesic equation of $\sigma_{2}$ in $M$, i.e. $\sigma_{2}^{\prime \prime}=A_{M}\left(\sigma_{2}^{\prime}, \sigma_{2}^{\prime}\right)$, where $A_{M}$ is the second fundamental form of the embedding of $M$ in $\mathbb{R}^{N}$, and by (M1) in the Section 1 ,

$$
\left|\sigma_{2}^{\prime \prime}\right| \leq\left(\sup _{M}\left|A_{M}\right|\right)\left|\sigma_{2}^{\prime}\right|^{2} \leq \frac{1}{16}\left|\sigma_{2}^{\prime}\right|^{2} \leq \frac{1}{16} L^{2}
$$


Since $\sigma_{2}^{\prime \prime}$ is normal to $M$, we can use Lemma 2.2 for $x=\sigma_{1}(s) \in M$ and $y=\sigma_{2}(s) \in M, s \in I$, hence

$$
\begin{aligned}
\left|\int_{I}\left\langle\left(\sigma_{1}-\sigma_{2}\right), \sigma_{2}^{\prime \prime}\right\rangle d s\right| & \leq \int_{I} \frac{1}{8}\left|\sigma_{2}^{\prime \prime}\right| \cdot\left|\sigma_{1}-\sigma_{2}\right|^{2} d s \leq \frac{1}{8} \cdot \frac{L^{2}}{16} \int_{I}\left|\sigma_{1}-\sigma_{2}\right|^{2} d s \\
& \leq \frac{1}{128} L^{2} \cdot \frac{l^{2}}{2} \int_{I}\left|\sigma_{1}^{\prime}-\sigma_{2}^{\prime}\right|^{2} d s \leq \frac{1}{8} \int_{I}\left|\sigma_{1}^{\prime}-\sigma_{2}^{\prime}\right|^{2} d s
\end{aligned}
$$

where we used the modified Wirtinger inequality (c.f. Lemma 2.1) in the third " $\leq$ ".

Now plug (3.5) (3.6) into (3.3),

$$
\int_{I}\left|\sigma_{1}^{\prime}-\sigma_{2}^{\prime}\right|^{2} d s \leq 2\left(\int_{I}\left|\sigma_{1}^{\prime}\right|^{2} d s-\int_{I}\left|\sigma_{2}^{\prime}\right|^{2} d s\right) .
$$

Applying the modified Wirtinger inequality (c.f. Lemma 2.1) with the above inequality, we can get (3.2).

Now we can finish the proof of Property (3).

Proof. (of Property (3) of $\Psi$ ) For Step 1 of $\Psi$, apply Lemma 3.2 on the boundary intervals $\left[0, x_{2}\right]$ and $\left[x_{2 L-2}, 1\right]$, and use Lemma 3.1 on the inner intervals $\left[x_{2 j}, x_{2 j+2}\right], j=1, \cdots, L-2$, and sum them together, we have

$$
\operatorname{dist}^{2}\left(\gamma, \gamma_{e}\right) \leq C\left(E(\gamma)-E\left(\gamma_{e}\right)\right) \leq C^{\prime}\left(\frac{\operatorname{Length}^{2}(\gamma)-\operatorname{Length}^{2}\left(\gamma_{e}\right)}{\operatorname{Length}^{2}\left(\gamma_{e}\right)}\right)
$$

where in the last " $\leq$ " we used the fact that $E(\gamma)=\operatorname{Length}^{2}(\gamma)$ as $\gamma$ has constant speed, and $\operatorname{Length}^{2}\left(\gamma_{e}\right) \leq E\left(\gamma_{e}\right)$, and Length $\left(\gamma_{e}\right) \leq \operatorname{Length}(\gamma) \leq L$.

The bound of $\operatorname{dist}\left(\gamma_{e}, \tilde{\gamma}_{e}\right)$ is similar to that in [CMM08, CM11, but as different parametrization is used here, we will give the details for completeness. In fact, $\gamma_{e}$ can be viewed as a reparametrization of $\tilde{\gamma}_{e}$, i.e. $\gamma_{e}=\tilde{\gamma}_{e} \circ P$, where $P: I \rightarrow I$ is a monotone piecewise linear map. Since $\tilde{\gamma}_{e}$ has constant speed, $\left|\tilde{\gamma}_{e}^{\prime}\right|=\operatorname{Length}\left(\tilde{\gamma}_{e}\right) / 1=\operatorname{Length}\left(\tilde{\gamma}_{e}\right)$. Also $\int_{I} P^{\prime} d s=1$, hence

$$
\begin{aligned}
\int_{I}\left(P^{\prime}-1\right)^{2} d s & =\int_{I}\left|P^{\prime}\right|^{2} d s-1=\int_{I}\left(\frac{\left|\gamma_{e}^{\prime}\right|}{\left|\tilde{\gamma}_{e}^{\prime} \circ P\right|}\right)^{2} d s-1 \\
& =\frac{1}{\operatorname{Length}^{2}\left(\tilde{\gamma}_{e}\right)} \int_{I}\left|\gamma_{e}^{\prime}\right|^{2} d s-1=\frac{E\left(\gamma_{e}\right)-\operatorname{Length}^{2}\left(\tilde{\gamma}_{e}\right)}{\operatorname{Length}^{2}\left(\tilde{\gamma}_{e}\right)} \\
& \leq \frac{\text { Length }^{2}(\gamma)-\text { Length }^{2}\left(\tilde{\gamma}_{e}\right)}{\operatorname{Length}^{2}\left(\tilde{\gamma}_{e}\right)}
\end{aligned}
$$

where in the last " $\leq$ " we used the fact that $E\left(\gamma_{e}\right) \leq E(\gamma)=\operatorname{Length}^{2}(\gamma)$ as $\gamma$ has constant speed.

To bound $\operatorname{dist}\left(\gamma_{e}, \tilde{\gamma}_{e}\right)$, as $\gamma_{e}(0)=\tilde{\gamma}_{e}(0)$ and $\gamma_{e}(1)=\tilde{\gamma}_{e}(1)$, we can combine the Wirtinger inequality with the following estimate,

$$
\begin{aligned}
\int_{I}\left|\gamma_{e}^{\prime}-\tilde{\gamma}_{e}^{\prime}\right|^{2} d s & =\int_{I}\left|\left(\tilde{\gamma}_{e}^{\prime} \circ P\right) P^{\prime}-\tilde{\gamma}_{e}^{\prime}\right|^{2} d s \\
& \leq 2 \int_{I}\left|\left(\tilde{\gamma}_{e}^{\prime} \circ P\right) P^{\prime}-\tilde{\gamma}_{e}^{\prime} \circ P\right|^{2} d s+2 \int_{I}\left|\tilde{\gamma}_{e}^{\prime} \circ P-\tilde{\gamma}_{e}^{\prime}\right|^{2} d s
\end{aligned}
$$


For the first term, using the fact that $\tilde{\gamma}_{e}$ has constant speed, we have $\left|\tilde{\gamma}_{e}^{\prime}\right| \leq L$, hence

$$
\int_{I}\left|\left(\tilde{\gamma}_{e}^{\prime} \circ P\right) P^{\prime}-\tilde{\gamma}_{e}^{\prime} \circ P\right|^{2} d s \leq \sup _{I}\left|\tilde{\gamma}_{e}^{\prime}\right|^{2} \cdot \int_{I}\left|P^{\prime}-1\right|^{2} d s \leq L^{2} \int_{I}\left|P^{\prime}-1\right|^{2} d s .
$$

For the second term, using the fact that $\tilde{\gamma}_{e}$ is a piecewise linear map, $\left|\tilde{\gamma}_{e}^{\prime \prime}\right|=\left|A_{M}\left(\tilde{\gamma}_{e}^{\prime}, \tilde{\gamma}_{e}^{\prime}\right)\right| \leq \frac{L^{2}}{16}$ (using property (M1) in Section 1) away from break points. So if $x, y \in I$ are not separated by a break point of $\tilde{\gamma}_{e}$, then by the fundamental theorem of calculus,

$$
\left|\tilde{\gamma}_{e}^{\prime}(x)-\tilde{\gamma}_{e}^{\prime}(y)\right| \leq \frac{L^{2}}{16}|x-y| .
$$

Now divide $[0,1]$ into two sets $I_{1}$ and $I_{2}$, where $I_{1}$ is the set of points within distance $\left(\int_{I} \mid P^{\prime}-\right.$ $\left.\left.1\right|^{2}\right)^{1 / 2}$ of a break point for $\tilde{\gamma}_{e}$, so $\operatorname{Length}\left(I_{2}\right)=L \cdot\left(2\left(\int_{I}\left|P^{\prime}-1\right|^{2}\right)^{1 / 2}\right)=2 L\left(\int_{I}\left|P^{\prime}-1\right|^{2}\right)^{1 / 2}$. As $P(0)=0$, we have

$$
|P(x)-x| \leq\left|\int_{0}^{x}\left(P^{\prime}(s)-1\right) d s\right| \leq x^{1 / 2}\left(\int_{I}\left|P^{\prime}-1\right|^{2}\right)^{1 / 2} \leq\left(\int_{I}\left|P^{\prime}-1\right|^{2}\right)^{1 / 2} .
$$

So if $x \in I_{2}$, then $P(x)$ lies in the same geodesic segment as $x$. Hence using (3.11) and the Wirtinger inequality,

$$
\int_{I_{2}}\left|\tilde{\gamma}_{e}^{\prime} \circ P-\tilde{\gamma}_{e}^{\prime}\right|^{2} d s \leq \frac{L^{4}}{256} \int_{I_{2}}|P(s)-s|^{2} d s \leq \frac{L^{4}}{256} \int_{I}\left|P^{\prime}-1\right|^{2} d s .
$$

Also as $\left|\tilde{\gamma}_{e}^{\prime}\right| \leq L$, so on $I_{1}$,

$$
\int_{I_{1}}\left|\tilde{\gamma}_{e}^{\prime} \circ P-\tilde{\gamma}_{e}^{\prime}\right|^{2} d s \leq 4 L^{2} \operatorname{Length}\left(I_{2}\right) \leq 8 L^{3}\left(\int_{I}\left|P^{\prime}-1\right|^{2}\right)^{1 / 2} .
$$

Combining the above two inequalities with (3.10) (3.9) and (3.8), together with (3.7), we can prove property (3) for Step 1 and Step 2 by realizing that $\operatorname{Length}\left(\gamma_{e}\right), \operatorname{Length}\left(\tilde{\gamma}_{e}\right) \geq$ $\operatorname{Length}(\Psi(\gamma))$. Estimates for $\operatorname{dist}\left(\tilde{\gamma}_{e}, \gamma_{o}\right)$ and $\operatorname{dist}\left(\gamma_{o}, \tilde{\gamma}_{o}\right)$ in Step 3 and Step 4 are the same and even easier as we only need Lemma 3.1, so we omit the proof here.

3.3 Property (4) of $\Psi$. The fact that fixed points of $\Psi$ are geodesics in the case of closed curve was discussed in [CM11, page 169] Cr88, §2].

Lemma 3.3. Given $\gamma \in \Lambda$, if $\Psi(\gamma)=\gamma$, then $\gamma \in G$, i.e. $\gamma$ is a geodesic with free boundary on $N$.

Proof. We can assume that $\gamma$ is not a point curve, or the statement is trivial. By the discussion at the end of 3.1 , the energy is non-increasing under the four steps, i.e. $E(\gamma) \geq E\left(\gamma_{e}\right) \geq$ $E\left(\tilde{\gamma}_{e}\right) \geq E\left(\gamma_{o}\right) \geq E(\Psi(\gamma))$. As $E(\Psi(\gamma))=E(\gamma)$, the energy must be the same. For $\gamma_{e} \rightarrow \tilde{\gamma}_{e}$ and $\gamma_{o} \rightarrow \tilde{\gamma}_{o}=\Psi(\gamma)$, by (3.8) where $E\left(\gamma_{e}\right)=E\left(\tilde{\gamma}_{e}\right)=$ Length $^{2}\left(\tilde{\gamma}_{e}\right)$ (similar estimates also hold for $\gamma_{o}$ and $\tilde{\gamma}_{o}$ ), we know that $\gamma_{e}=\tilde{\gamma}_{e}$ and $\gamma_{o}=\Psi(\gamma)=\gamma$, i.e. the reparametrization $P \equiv i d$. As $E(\gamma)=E\left(\gamma_{e}\right)=E\left(\gamma_{o}\right)$, by Lemma 3.1 and Lemma 3.2, we know that $\gamma=\gamma_{e}=\gamma_{o}$. The fact $\gamma=\gamma_{e}$ implies that the break points of $\gamma$ can only appear at $\gamma\left(x_{2 j}\right), j=1, \cdots, L-1$, and $\gamma$ is perpendicular to $N$ at boundary points, i.e. $\gamma$ has free boundary on $N$. Also as the points $x_{2 j}, j=1, \cdots, L-1$ are smooth points of $\gamma_{o}$, hence $\gamma$ has no break points. 
3.4 Property (5) of $\Psi$. This is a direct corollary of other properties of $\Psi$ by a contradiction argument. Similar argument in the case of closed curves appeared in [CM11, page 169]. Suppose in contradiction, say there exists an $\epsilon>0$, and a sequence of $\gamma_{j} \in \Lambda$, such that

$$
\operatorname{Length}\left(\Psi\left(\gamma_{j}\right)\right) \geq \operatorname{Length}\left(\gamma_{j}\right)-\frac{1}{j}, \quad \operatorname{dist}\left(\gamma_{j}, G\right) \geq \epsilon .
$$

As the second condition implies that $\operatorname{Length}\left(\gamma_{j}\right)$ is bounded away from zero, the first condition and property (3) implies that

$$
\operatorname{dist}\left(\gamma_{j}, \Psi\left(\gamma_{j}\right)\right) \rightarrow 0
$$

Note that the space $\Lambda$ is compact, as every $\sigma \in \Lambda$ depends continuously on the boundary points on $N$ and the $L-1$ break points in the compact manifold $M$ (or in a compact convex region when $M$ is non-compact). Hence a subsequence of $\left\{\gamma_{j}\right\}$ will converge to some $\gamma \in \Lambda$. As $\Psi$ is continuous on $\Lambda$, i.e. property (1), $\Psi(\gamma)=\gamma$, which implies that $\gamma \in G$ by Lemma 3.3. contradiction to that $\operatorname{dist}(\gamma, G)=\lim _{j \rightarrow \infty} \operatorname{dist}\left(\gamma_{j}, G\right) \geq \epsilon$.

3.5 Property (1) of $\Psi$. Now we prove the continuity of $\Psi$. Similar argument in the case of closed curves appeared in [CM11]. Here we need to carefully deal with the case of free boundary linear replacement. The continuity of the first two steps for $\Psi$ follows from the following lemma, and the continuity of the last two steps follows from the closed case (c.f. CM11, Lemma 5.3]).

Lemma 3.4. Given a large integer L. Let $\gamma:[0,1] \rightarrow M$ be a $W^{1,2}$-map, with $\gamma(0), \gamma(1) \in N$, $E(\gamma) \leq L$. If $\gamma_{e}, \tilde{\gamma}_{e}$ are given by the first two steps for $\Psi$ in 3.1 , then the map $\gamma \rightarrow \tilde{\gamma}_{e}$ is continuous from $W^{1,2}(I, M)$ to $\Lambda$.

Recall that $\left\{x_{0}, x_{2} \cdots, x_{2 L-2}, x_{2 L}=1\right\}$ are the evenly spaced points where we do linear replacement. By (2.1), $\left|\gamma\left(x_{2 j}\right)-\gamma\left(x_{2 j+2}\right)\right| \leq\left(\frac{1}{L} E(\gamma)\right)^{1 / 2} \leq 1$, hence $d_{M}\left(\gamma\left(x_{2 j}\right), \gamma\left(x_{2 j+2}\right)\right) \leq 2$ by (M3) in \$1 and we can apply Step 1 of 3.1 by (M2) and (M2) in \$1. Now recall two observations used in [CM11] $((\mathrm{C} 1)(\mathrm{C} 2)$ already appeared in [CM11, page 170]),

(C1) Curves which are $W^{1,2}$ close are also $C^{0}$ close , hence the points $\gamma_{e}\left(x_{2 j}\right)=\gamma\left(x_{2 j}\right)$ are continuous with respect to $W^{1,2}$-norm of $\gamma$.

(C2) Let $\Gamma=\left\{(x, y) \in M \times M: \operatorname{dist}_{M}(x, y) \leq 4\right\}$, and define

$$
H: \Gamma \rightarrow C^{1}\left(\left[0, \frac{1}{L}\right], M\right)
$$

such that $H(x, y)$ is the linear map from $x$ to $y$, then $H$ is continuous on $\Gamma$.

$(\mathrm{C} 2)^{\prime}$ Let $\Gamma^{\prime}=\left\{x \in M: \operatorname{dist}_{M}(x, N) \leq 2\right\}$, and define

$$
H^{\prime}: \Gamma^{\prime} \rightarrow C^{1}\left(\left[0, \frac{1}{L}\right], M\right)
$$

such that $H^{\prime}(x)$ is the minimizing geodesic from $x$ to $N$, then $H^{\prime}$ is continuous on $\Gamma^{\prime}$. 
To make $(\mathrm{C} 2)^{\prime}$ precise, we have the following lemma.

Lemma 3.5. Given $L \geq 2$, and $x_{1}, x_{2} \in \Gamma^{\prime}$, and let $\sigma_{1}, \sigma_{2}:\left[0, \frac{1}{L}\right] \rightarrow M$ be two minimizing geodesic from $\sigma_{1}(0)=x_{1}$ and $\sigma_{2}(0)=x_{2}$ to $N$ respectively, with $\sigma_{1}\left(\frac{1}{L}\right), \sigma_{2}\left(\frac{1}{L}\right) \in N$, and meet $N$ orthogonally there, then there exists a continuous function $\phi:[0, \infty) \rightarrow[0, \infty)$, with $\phi(0)=0$, such that

$$
\operatorname{dist}\left(\sigma_{1}, \sigma_{2}\right) \leq \phi\left(d_{M}\left(x_{1}, x_{2}\right)\right) .
$$

Proof. Using integration by parts as in Lemma 3.2

$$
\begin{aligned}
\int\left|\sigma_{1}^{\prime}\right|^{2} & -\int\left|\sigma_{2}^{\prime}\right|^{2}-\int\left|\sigma_{1}^{\prime}-\sigma_{2}^{\prime}\right|^{2}=2 \int\left\langle\sigma_{2}^{\prime},\left(\sigma_{1}-\sigma_{2}\right)^{\prime}\right\rangle d t \\
& =2 \underbrace{\left\langle\sigma_{2}^{\prime}\left(\frac{1}{L}\right), \sigma_{1}\left(\frac{1}{L}\right)-\sigma_{2}\left(\frac{1}{L}\right)\right\rangle}_{I_{1}}-2 \underbrace{\left\langle\sigma_{2}^{\prime}(0), \sigma_{1}(0)-\sigma_{2}(0)\right\rangle}_{I_{2}}-2 \underbrace{\int\left\langle\left(\sigma_{1}-\sigma_{2}\right), \sigma_{2}^{\prime \prime}\right\rangle}_{I_{3}} .
\end{aligned}
$$

First using the fact that $\left|\sigma_{2}^{\prime}\right|=\operatorname{Length}\left(\sigma_{2}\right)=d_{M}\left(x_{2}, N\right) \leq 2$,

$$
\left|I_{2}\right| \leq\left|\sigma_{2}^{\prime}(0)\right| \cdot\left|\left(\sigma_{1}-\sigma_{2}\right)(0)\right| \leq 2 d_{M}\left(x_{1}, x_{2}\right) .
$$

Also using Lemma 2.2 and the fundamental theorem of calculus,

$$
\begin{aligned}
\left|I_{1}\right| & \leq \frac{1}{8}\left|\sigma_{2}^{\prime}\left(\frac{1}{L}\right)\right| \cdot\left|\sigma_{1}\left(\frac{1}{L}\right)-\sigma_{2}\left(\frac{1}{L}\right)\right|^{2} \\
& \leq \frac{1}{8} d_{M}\left(x_{2}, N\right) \cdot\left|\sigma_{1}(0)-\sigma_{2}(0)+\int_{0}^{1 / L}\left(\sigma_{1}^{\prime}-\sigma_{2}^{\prime}\right) d s\right|^{2} \\
& \leq \frac{1}{8} d_{M}\left(x_{2}, N\right) \cdot 2\left[\left|x_{1}-x_{2}\right|^{2}+\left|\int_{0}^{1 / L}\left(\sigma_{1}^{\prime}-\sigma_{2}^{\prime}\right) d s\right|^{2}\right] \\
& \leq \frac{1}{8} d_{M}\left(x_{2}, N\right) \cdot 2\left[\left|x_{1}-x_{2}\right|^{2}+\frac{1}{L} \int_{0}^{1 / L}\left|\sigma_{1}^{\prime}-\sigma_{2}^{\prime}\right|^{2} d s\right] \\
& \leq \frac{1}{2} d_{M}^{2}\left(x_{1}, x_{2}\right)+\frac{1}{4} \int_{0}^{1 / L}\left|\sigma_{1}^{\prime}-\sigma_{2}^{\prime}\right|^{2} d s,
\end{aligned}
$$

where we used the Cauchy-Schwartz inequality in the fourth " $\leq$ ", and the fact that $d_{M}\left(x_{2}, N\right) \leq$ 2 and $L \geq 2$ in the last " $\leq$ ".

Similarly to (3.6),

$$
\begin{aligned}
\left|I_{3}\right| & \leq \int_{0}^{1 / L}\left|\left\langle\sigma_{1}-\sigma_{2}, \sigma_{2}^{\prime \prime}\right\rangle\right| d t \leq \int_{0}^{1 / L} \frac{1}{8}\left|\sigma_{2}^{\prime \prime}\right| \cdot\left|\sigma_{1}-\sigma_{2}\right|^{2} d t \\
& \leq \frac{1}{8}\left(\sup _{M}\left|A_{M}\right|\right) \cdot\left|\sigma_{2}^{\prime}\right|^{2} \int_{0}^{1 / L}\left|\sigma_{1}-\sigma_{2}\right|^{2} d t \\
& \leq \frac{1}{32} \int_{0}^{1 / L}\left|\left(\sigma_{1}-\sigma_{2}\right)(0)+\int_{0}^{t}\left(\sigma_{1}^{\prime}-\sigma_{2}^{\prime}\right)(s) d s\right|^{2} d t \\
& \leq \frac{1}{16} \int_{0}^{1 / L}\left[\left|\sigma_{1}(0)-\sigma_{2}(0)\right|^{2}+t \int_{0}^{t}\left|\sigma_{1}^{\prime}-\sigma_{2}^{\prime}\right|^{2}(s) d s\right] d t \\
& \leq \frac{1}{16 L} d_{M}^{2}\left(x_{1}, x_{2}\right)+\frac{1}{32 L} \int_{0}^{1 / L}\left|\sigma_{1}^{\prime}-\sigma_{2}^{\prime}\right|^{2}(t) d t
\end{aligned}
$$


By plugging the above to (3.14), we get

$$
E\left(\sigma_{1}\right)-E\left(\sigma_{2}\right)+\left(1+\frac{1}{8 L}\right) d_{M}^{2}\left(x_{1}, x_{2}\right)+4 d_{M}\left(x_{1}, x_{2}\right) \geq c \int\left|\sigma_{1}^{\prime}-\sigma_{2}^{\prime}\right|^{2} d s,
$$

for some $c>0$. Reverse the role of $\sigma_{1}$ and $\sigma_{2}$, and sum the above inequality together, we get

$$
\varphi\left(d_{M}\left(x_{1}, x_{2}\right)\right) \geq c \int\left|\sigma_{1}^{\prime}-\sigma_{2}^{\prime}\right|^{2} d s
$$

for $\varphi(x)=\left(1+\frac{1}{8 L}\right) x^{2}+4 x$.

Similarly by the fundamental theorem of calculus,

$$
\begin{aligned}
\int\left|\sigma_{1}-\sigma_{2}\right|^{2} & \leq \int\left|\left[\sigma_{1}(0)-\sigma_{2}(0)+\int_{0}^{s}\left(\sigma_{1}^{\prime}-\sigma_{2}^{\prime}\right) d t\right]\right|^{2} d s \\
& \leq \frac{2}{L} d_{M}^{2}\left(x_{1}, x_{2}\right)+\frac{1}{L} \int\left|\sigma_{1}^{\prime}-\sigma_{2}^{\prime}\right|^{2}
\end{aligned}
$$

Remark 3.6. The lemma is still true if the defining intervals of $\sigma$ has length less than $\frac{1}{L} \leq \frac{1}{2}$. To get $(\mathrm{C} 2)^{\prime}$, we can use the above lemma and the fundamental theorem of calculus to show that $d_{M}\left(\sigma_{1}\left(\frac{1}{L}\right), \sigma_{2}\left(\frac{1}{L}\right)\right)$ depends continuously on $d_{M}\left(\sigma_{1}(0), \sigma_{2}(0)\right)$, and then use $(\mathrm{C} 2)$.

Proof. (of Lemma 3.4) Given two $W^{1,2}$-maps $\gamma^{1}, \gamma^{2}$ as in the lemma which are $W^{1,2}$ close, we can assume that $\gamma^{1}$ is not a constant curve, or the proof is trivial. Let $a_{0}^{i}=d_{M}\left(\gamma^{i}\left(x_{2}\right), N\right)$, $a_{L-1}^{i}=d_{M}\left(\gamma^{i}\left(x_{2 L-2}\right), N\right)$, and $a_{j}^{i}=d_{M}\left(\gamma^{i}\left(x_{2 j}\right), \gamma^{i}\left(x_{2 j+2}\right)\right), j=1, \cdots, L-2$, and $S^{i}=$ $\sum_{j=0}^{L-1} a_{j}^{i}, i=1,2$, then $S^{i}>0$. By $(\mathrm{C} 1)$, the points $\gamma^{i}\left(x_{2 j}\right)=\gamma_{e}^{i}\left(x_{2 j}\right), j=1, \cdots, L-1$ and hence the numbers $a_{j}^{i}, S^{i}$ are all continuous with respect to $\gamma^{i}$ for $i=1,2$. Therefore, the geodesic segments $\left.\gamma_{e}^{1}\right|_{\left[x_{2 j}, x_{2 j+2}\right]}$ and $\left.\gamma_{e}^{2}\right|_{\left[x_{2 j}, x_{2 j+2}\right]}$ are $C^{1}$ close on $\left[x_{2 j}, x_{2 j+2}\right]$ for $j=0,1, \cdots, L-$ 1 by $(\mathrm{C} 2)$ and $(\mathrm{C} 2)^{\prime}$, so $\gamma \rightarrow \gamma_{e}$ is continuous.

Since the reparametrization from $\gamma_{e} \rightarrow \tilde{\gamma}_{e}$ fixes the boundary point, $\tilde{\gamma}_{e}^{1}(0)$ and $\tilde{\gamma}_{e}^{2}(0)$ are close by Remark 3.6. To show that $\tilde{\gamma}_{e}^{1}$ and $\tilde{\gamma}_{e}^{2}$ are $W^{1,2}$ close, we only need to show that $\int\left|\left(\tilde{\gamma}_{e}^{1}-\tilde{\gamma}_{e}^{2}\right)^{\prime}\right|^{2}$ is small, and then apply the modified Wirtinger inequality to $\left(\tilde{\gamma}_{e}^{1}(t)-\tilde{\gamma}_{e}^{2}(t)-\right.$ $\left.\left[\tilde{\gamma}_{e}^{1}(0)-\tilde{\gamma}_{e}^{2}(0)\right]\right)$.

After the reparametrization, the constant speed curves $\tilde{\gamma}_{e}^{i}$ are geodesic segments on the intervals $I_{j}^{i}=\left[\frac{1}{S^{2}} \sum_{l<j} a_{l}^{i}, \frac{1}{S^{2}} \sum_{l \leq j} a_{l}^{i}\right]$, and $\tilde{\gamma}_{e}^{i}=\gamma_{e} \circ P_{j}^{i}$ on each interval $I_{j}^{i}$ with $a_{j}^{i} \neq 0$, where the reparametrization $P_{j}^{i}: I_{j}^{i} \rightarrow\left[x_{2 j}, x_{2 j+2}\right]$ are just linear maps. Clearly $I_{j}^{1}, I_{j}^{2}$ and $P_{j}^{1}, P_{j}^{2}$ are close respectively. Let $I_{j}=I_{j}^{1} \cap I_{j}^{2}$, then $I \backslash\left\{\cup_{j=0}^{L-1} I_{j}\right\}$ is very small. Using the fact that $\tilde{\gamma}_{e}^{i}$ have constant speed with energy less than $L$, we have $\left|\left(\tilde{\gamma}_{e}^{i}\right)^{\prime}\right| \leq L$, so

$$
\int_{I \backslash \cup I_{j}}\left|\left(\tilde{\gamma}_{e}^{1}-\tilde{\gamma}_{e}^{2}\right)^{\prime}\right|^{2} \leq 4 L^{2} \cdot \operatorname{Length}\left(I \backslash \cup_{j} I_{j}\right),
$$

which is hence small. 
Given $\epsilon>0$ small enough, we can divide $I_{j}$ into two sub-classes. If $a_{j}^{1}<\epsilon$, we can assume that $a_{j}^{2}<2 \epsilon$ by continuity, then

$$
\int_{I_{j}}\left|\left(\tilde{\gamma}_{e}^{1}-\tilde{\gamma}_{e}^{2}\right)^{\prime}\right|^{2} \leq 2 \int_{I_{j}^{1}}\left|\left(\tilde{\gamma}_{e}\right)^{\prime}\right|^{2}+2 \int_{I_{j}^{2}}\left|\left(\tilde{\gamma}_{e}\right)^{\prime}\right|^{2} \leq 2 L^{2}\left(a_{j}^{1}+a_{j}^{2}\right)<6 L^{2} \epsilon
$$

If $a_{j}^{1} \geq \epsilon$, we can assume that $a_{j}^{2} \geq \frac{\epsilon}{2}$ by continuity, then $\left|\left(P_{j}^{i}\right)^{\prime}\right|=\frac{S^{i}}{a_{j}^{i}}$, and

$$
\int_{I_{j}}\left|\left(\tilde{\gamma}_{e}^{1}-\tilde{\gamma}_{e}^{2}\right)^{\prime}\right|^{2}=\int_{I_{j}}\left|\left(\left(\gamma_{e}^{1}\right)^{\prime} \circ P_{j}^{1}\right)\left(P_{j}^{1}\right)^{\prime}-\left(\left(\gamma_{e}^{2}\right)^{\prime} \circ P_{j}^{2}\right)\left(P_{j}^{2}\right)^{\prime}\right|^{2}
$$

which can be made small as $\gamma_{e}^{i}$ are close in $C^{1}$-norm and bounded in $C^{2}$-norm 4 , and $P_{j}^{i},\left|\left(P_{j}^{i}\right)^{\prime}\right|$ are close as linear maps and numbers respectively.

3.6 Property (2) of $\Psi$. We only need to prove that $\Psi$ preserves the homotopy class, as the length decreasing property is trivially true. In fact, this is just a corollary of Lemma 4.1 and Remark 4.2 in the next section.

\section{Good sweepouts and free boundary min-max geodesics}

In this section, we will discuss apply the curve shortening process $\Psi$ to sweepouts in Definition 1.2 and prove Theorem 1.6. The foremost interesting question is when the width $W$, defined by (1.1) corresponding to a sweepout $\sigma_{0}$, is positive. In fact, $W$ is positive when $\sigma_{0}$ represents a nontrivial homotopy class in $\Omega$. A special case is when the constraint submanifold $N$ bounds a non-contractable disk (among all disks with boundary lying on $N$ ). Suppose in contradiction that the width is small enough, then there exists a sweepout $\sigma$ homotopic to $\sigma_{0}$, and the energy of each $\sigma(t, \cdot)$ is very small, hence $\sigma(t, \cdot)$ all lie in a convex geodesic neighborhood of $N$. Property (M2)' implies that we can continuously shrink $\sigma(t, \cdot)$ to a point through curves with boundary lying on $N$. In fact, we can shrink $\sigma(\cdot, t)$ in a continuous way with respect to " $t$ " (e.g. using local coordinate charts), and homotopically deform $\sigma$ to a family of point curves, which contradicts the fact that $\sigma_{0}$ is homotopically nontrivial.

4.1 Sweepouts and $\Psi$. Given a sweepout $\hat{\sigma} \in \Omega$, we need to define a precise way to apply the curve shortening process $\Psi$ to deform $\hat{\sigma}$ to a sweep-out in $\Lambda$. Assume that the maximal energy of slices $\hat{\sigma}(t)(\cdot)=\hat{\sigma}(t, \cdot)$ is bounded by a number $W_{0}$, i.e. $\max _{t \in[0,1]} E(\hat{\sigma}(t)) \leq W_{0}$, then the Cauchy-Schwartz inequality implies a uniform bound for the length and $C^{1 / 2}$-Hölder continuity for each $\hat{\sigma}(t)$, i.e.

$$
d_{M}(\hat{\sigma}(t, x), \hat{\sigma}(t, y)) \leq \operatorname{Length}\left(\left.\hat{\sigma}(t)\right|_{[x, y]}\right)=\int_{x}^{y}\left|\partial_{x} \hat{\sigma}(t, x)\right| d x
$$

\footnotetext{
${ }^{4}$ This is because $\left|\gamma_{e}^{\prime \prime}\right| \leq \sup \left|A_{M}\right| \cdot\left|\gamma_{e}^{\prime}\right|^{2} \leq \frac{1}{16} L^{2}$ by the geodesic equation and (M1) in $₫ 1$
} 


$$
\leq|y-x|^{\frac{1}{2}}\left(\int_{I}\left|\partial_{x} \hat{\sigma}(t, x)\right|^{2} d x\right)^{\frac{1}{2}} \leq|x-y|^{\frac{1}{2}} W_{0}^{\frac{1}{2}} .
$$

We will deform $\hat{\sigma}(t)$ to $\sigma(t)$ by Step 1 and Step 2 in 33.1 , By the uniform $C^{1 / 2}$-Hölder bound, there exists an evenly spaced partition of $I=[0,1]$ by $N$ points, i.e. $x_{0}=0, x_{1}, x_{2}, \cdots, x_{N}=1$, such that the length of $\left.\hat{\sigma}(t)\right|_{\left[x_{j}, x_{j+1}\right]}$ is bounded by 2 for all $t \in[0,1], j=0,1, \cdots, N-1$. Hence we can apply Step 1 to $\hat{\sigma}(t)$ by (M2) and (M2) to get $\sigma_{e}$, and then reparametrize $\sigma_{e}(t)$ to get a constant speed mapping $\tilde{\sigma}_{e}(t)$, which we denote by $\sigma(t)$. By Lemma 3.4, we know that $t \rightarrow \sigma(t)$ is a continuous mapping from $[0,1]$ to $W^{1,2}(I, M)$, and it is easy to see that $\sigma \in \Omega$. Also the length bound of $\hat{\sigma}(t)$ implies a uniform Lipschitz bound of $\sigma(t)$ by $W_{0}^{1 / 2}$, as $\sigma(t)$ has constant speed and shorter than $\hat{\sigma}(t)$. Hence $\sigma(t) \in \Lambda$ for some $L \in \mathbb{N}$, with $L$ larger than $N$ and $W_{0}^{1 / 2}$, so $\sigma(t)$ is a continuous path in $\Lambda$. In the next lemma, we show that $\hat{\sigma}$ and $\sigma$ are homotopic.

Lemma 4.1. Given $\hat{\sigma} \in \Omega$, and $\sigma_{e}, \tilde{\sigma}_{e}$ as above, then $\hat{\sigma}, \sigma_{e}$ and $\tilde{\sigma}_{e}$ are all homotopic in $\Omega$.

Proof. First we show that $\hat{\sigma}$ is homotopic to $\sigma_{e}$ in $\Omega$. Corresponding the $L$ break point 5 : $x_{0}=0, x_{1}, \cdots, x_{L}=1$, we deform $\hat{\sigma}$ to $\sigma_{e}$ in $L$ steps. For $s \in\left[0, x_{1}\right]$, define $F:[0,1] \times I \times$ $\left[0, x_{1}\right] \rightarrow M$, such that

$$
F(t, x, s)=\left\{\begin{array}{l}
H^{\prime}(\hat{\sigma}(t, s))(x), \quad \text { if } 0 \leq x \leq s \\
\hat{\sigma}(t, x), \quad \text { if } s \leq x \leq 1
\end{array}\right.
$$

where $H^{\prime}$ is given by (3.13) 6 . $(\mathrm{C} 2)^{\prime}$ and Lemma 3.5 imply that $F$ is continuous, hence $F(\cdot, \cdot s)$ lies in $\Omega$ by the definition of $H^{\prime}$. So $F$ defines homotopy between $\hat{\sigma}(t, x)=F(t, x, 0)$ with $\sigma_{1}(t, x)=F\left(t, x, x_{1}\right)$ in $\Omega$. Now we inductively define $F(t, x, s)$ as homotopy between $\sigma_{j}(t, x)$ and $\sigma_{j+1}(t, x)$ when $s \in\left[x_{j}, x_{j+1}\right], 1 \leq j \leq L-2$. Assume that $\sigma_{1}, \cdots, \sigma_{j}$, and $F(\cdot, \cdot, s)$ are well-defined for $s \in\left[0, x_{j}\right]$, where $F(\cdot, \cdot, s):\left[x_{l}, x_{l+1}\right] \rightarrow \Omega$ is a homotopy between $\sigma_{l}$ and $\sigma_{l+1}$, $1 \leq l \leq j-1$. For $s \in\left[x_{j}, x_{j+1}\right]$, define $F:[0,1] \times I \times\left[x_{j}, x_{j+1}\right] \rightarrow M$ by

$$
F(t, x, s)=\left\{\begin{array}{l}
H\left(\sigma_{j}\left(t, x_{j}\right), \sigma_{j}(t, s)\right)(x), \quad \text { if } x_{j} \leq x \leq s \\
\sigma_{j}(t, x), \quad \text { if } 0 \leq x \leq x_{j}, \text { or } s \leq x \leq 1
\end{array}\right.
$$

where $H$ is defined by (3.12) 7 . (C2) implies that $F$ is continuous, so $F(\cdot, \cdot, s) \in \Omega$, and $F$ defines a homotopy between $\sigma_{j}(t, x)=F\left(t, x, x_{j}\right)$ and $\sigma_{j+1}(t, x)=F\left(t, x, x_{j+1}\right)$. Finally, using similar argument, we can use $H^{\prime}$ to define a homotopy between $\sigma_{L-1}$ with $\sigma_{e}$ by $F$ : $[0,1] \times I \times\left[x_{L-1}, 1\right] \rightarrow M$, with

$$
F(t, x, s)=\left\{\begin{array}{l}
H^{\prime}\left(\sigma_{L-1}(t, s)\right)(x), \quad \text { if } x_{L-1}+1-s \leq x \leq 1 \\
\sigma_{L-1}(t, x), \quad \text { if } 0 \leq x \leq x_{L-1}+1-s
\end{array}\right.
$$

\footnotetext{
${ }^{5} L=N$ in the above case.

${ }^{6}$ Here the defining interval for $H^{\prime}(\cdot)$ is $[0, s]$, with $0 \leq s \leq x_{1}$.

${ }^{7}$ Here the defining interval for $H(\cdot, \cdot)$ is $\left[x_{j}, s\right]$, with $x_{j} \leq s \leq x_{j+1}$.
} 
Next we show that $\sigma_{e}$ is homotopic to $\tilde{\sigma}_{e}$ in $\Omega$. As $\tilde{\sigma}_{e}(t, \cdot)$ is a reparametrization of $\sigma_{e}(t, \cdot)$, we can write $\sigma_{e}(t, \cdot)=\tilde{\sigma}_{e}(t, \cdot) \circ P_{t}$, where $P_{t}:[0,1] \rightarrow[0,1]$ is a monotone piecewise linear map. By the proof of Lemma 3.4, $P_{t}$ depends continuously on " $t$ " 8 . Then

$$
G(t, x, s)=\tilde{\sigma}_{e}\left(t,(1-s) P_{t}(x)+s x\right),
$$

is a homotopy between $G(\cdot, \cdot, 0)=\sigma_{e}$ and $G(\cdot, \cdot, 1)=\tilde{\sigma}_{e}$. When $\operatorname{Length}\left(\gamma_{e}(t, \cdot)\right)=0$, we can let $P_{t}=i d$, and $G$ is well-defined.

Remark 4.2. Applying Step 3 and Step 4 of $\Psi$ in 3.1 to $\tilde{\sigma}_{e}(t)$ gives $\sigma_{o}(t)$ and $\tilde{\sigma}_{o}(t)$, with $\tilde{\sigma}_{o}(t) \in \Lambda$ for each $t$. $\sigma_{o}(t)$ an $\tilde{\sigma}_{o}(t)$ are both continuous mapping from $[0,1]$ to $W^{1,2}(I, M)$ by Property (1) of $\Psi$, and it is easily seen that $\tilde{\sigma}_{o}(t) \in \Omega$. The homotopy equivalence of $\tilde{\sigma}_{e}$, $\sigma_{o}$ and $\tilde{\sigma}_{o}$ in $\Omega$ follows from an easy modification of the above proof, as there is no boundary replacement here.

4.2 Almost maximal implies almost critical. We prove Theorem 1.6 in this section.

Proof. (Theorem [1.6) Take a sequence $\left\{\hat{\sigma}_{j}\right\} \subset\left[\sigma_{0}\right]$, such that

$$
\max _{t \in[0,1]} E\left(\hat{\sigma}_{j}(t)\right) \leq W_{0}+\frac{1}{j}
$$

By the discussion in 4 , we can deform $\hat{\sigma}_{j}$ to $\sigma_{j}$, such that $E\left(\sigma_{j}(t)\right) \leq E\left(\hat{\sigma}_{j}(t)\right), \sigma_{j}(t)$ is a continuous path in $\Lambda$, and $\sigma_{j}(t)$ is homotopic to $\hat{\sigma}_{j}(t)$ in $\Omega$ by Lemma 4.1, hence $\sigma_{j}(t) \in\left[\sigma_{0}\right]$. So $\max _{t \in[0,1]} E\left(\sigma_{j}(t)\right) \leq \max _{t \in[0,1]} E\left(\hat{\sigma}_{j}(t)\right) \leq W_{0}+\frac{1}{j}$.

Now apply the modified Birkhoff curve shortening map to each $\sigma_{j}(t)$ to get $\gamma_{j}(t)=$ $\Psi\left(\sigma_{j}(t)\right) \in \Lambda$, hence $\gamma_{j}(t)$ is homotopic to $\sigma_{j}(t)$ in $\Omega$ by Lemma 4.1 and Remark 4.2, so $\gamma_{j} \in\left[\sigma_{0}\right]$. As $E\left(\gamma_{j}(t)\right)=\operatorname{Length}^{2}\left(\gamma_{j}(t)\right) \leq \operatorname{Length}^{2}\left(\sigma_{j}(t)\right)=E\left(\sigma_{j}(t)\right)$,

$$
\max _{t \in[0,1]} E\left(\gamma_{j}(t)\right) \leq W_{0}+\frac{1}{j} .
$$

So $\lim _{j \rightarrow \infty} \max _{t \in[0,1]} E\left(\gamma_{j}(t)\right)=W_{0}=W\left(\left[\sigma_{0}\right]\right)$.

We will show that $\left\{\gamma_{j}\right\}$ satisfies the requirement of Theorem 1.6. If not, then there exist an $\epsilon>0$, a sequence $\delta_{i}>0$, with $\lim _{i \rightarrow \infty} \delta_{i}=0$, a subsequence $\gamma_{j_{i}}$, with $j_{i}>\frac{1}{\delta_{i}}$, and a sequence of $t_{i} \in[0,1]$, with $E\left(\gamma_{j_{i}}\left(t_{i}\right)\right)=\operatorname{Length}^{2}\left(\gamma_{j_{i}}\left(t_{i}\right)\right)>W_{0}-\delta_{i}$, but $\operatorname{dist}\left(\gamma_{j_{i}}\left(t_{i}\right), G\right) \geq \epsilon$. Also, as $E\left(\gamma_{j}\left(t_{i}\right)\right) \leq E\left(\sigma_{j}\left(t_{i}\right)\right) \leq W_{0}+\frac{1}{j_{i}} \leq W_{0}+\delta_{i}$, we have

$$
W_{0}-\delta_{i} \leq \operatorname{Length}^{2}\left(\gamma_{j_{i}}\left(t_{i}\right)\right) \leq \operatorname{Length}^{2}\left(\sigma_{j_{i}}\left(t_{i}\right)\right) \leq W_{0}+\delta_{i} .
$$

Now denote $\gamma_{i}=\gamma_{j_{i}}\left(t_{i}\right)$ and $\sigma_{i}=\sigma_{j_{i}}\left(t_{i}\right)$. Since $W_{0}>0$, then $\operatorname{Length}\left(\gamma_{i}\right) \geq \frac{\epsilon}{2}$ for $i$ large enough. Then Property (3) of $\Psi$ implies that $\operatorname{dist}\left(\gamma_{i}, \sigma_{i}\right) \leq \frac{\epsilon}{2}$ for $i$ large enough, as $\gamma_{i}=\Psi\left(\sigma_{i}\right)$, hence

$$
\operatorname{dist}\left(\sigma_{i}, G\right) \geq \operatorname{dist}\left(\gamma_{i}, G\right)-\operatorname{dist}\left(\gamma_{i}, \sigma_{i}\right) \geq \frac{\epsilon}{2} .
$$

\footnotetext{
$\left.{ }^{8} P_{t}\right|_{\left[x_{2 j}, x_{2 j+2}\right]}=\left(P_{j}(t)\right)^{-1}$ is a linear map from $\left[x_{2 j}, x_{2 j+2}\right]$ to $I_{j}(t)=\left[\frac{1}{S(t)} \sum_{l<j} a_{l}(t), \frac{1}{S(t)} \sum_{l \leq j} a_{l}(t)\right]$ using notations in the proof of Lemma 3.4
} 
But this is a contradiction to Property (5) of $\Psi$, as $\operatorname{Length}\left(\sigma_{i}\right)-\operatorname{Length}\left(\gamma_{i}\right) \rightarrow 0$ when $i \rightarrow \infty$.

\section{References}

[AF65] F. Almgren, The theory of varifolds, Mimeographed notes, Princeton, 1965.

[B] G. Birkhoff, Dynamical Systems, Colloq. Publ. IX, A.M.S., Providence, R. I. 1927.

[CD03] T. Colding and C. De Lellis, The min-max construction of minimal surfaces, Surveys in differential geometry, Vol. VIII (Boston, MA, 2002), 75-107, Int. Press, Somerville, MA, 2003.

[CMM08] T. Colding and W. Minicozzi II, Width and mean curvature flow, Geom. Topol. 12 (2008) 2517-2535.

[CMR08] T. Colding and W. Minicozzi II, Width and finite extinction time of Ricci flow, Geom. Topol. 12 (2008) 2537-2586.

[CM11] T. Colding and W. Minicozzi II, A course in minimal surfaces, Graduate Studies in Mathematics Volume 121, American Mathematical Society, 2011.

[Cr88] C. Croke, Area and the length of the shortest closed geodesic, J. Differential Geom. 27 (1988) $1-21$.

[F00] A. Fraser, On the free boundary variational problem for minimal disks, Comm. Pure Appl. Math. 53, 8 (2000), 931-971.

[Gr] M. Grayson, Shortening Embedded Curves, Ann. of Math, (2) 129, (1989), 71-111.

[Jo89] J. Jost, Embedded minimal surfaces in manifolds diffeomorphic to the three-dimensional ball or sphere, J. Differential Geom. 30, 2 (1989) 555-577.

[Kl82] W. Klingenberg, Riemannian geometry, de Gruyter, Berlin and New York, 1982.

[L14] M. Li, A general existence theorem of embedded minimal surfaces with free boundary, to appear in Comm. Pure Appl. Math. (2014).

[LW] L. Lin and L. Wang, Existence of Good Sweepouts on Closed Manifolds, Proc. Amer. Math. Soc. 138 (2010) 4081-4088.

[MN12] F. Marques and A. Neves, Min-max theory and the Willmore conjecture, Ann. of Math. (2) 179, (2014) 683-782.

[NR] A. Nabutovsky and R. Rotman, Linear bounds for lengths of geodesic loops on Riemannian 2spheres, J. Differential Geom. 89 (2011), 217-232.

[P81] J. Pitts, Existence and regularity of minimal surfaces on Riemannian manifold, Mathematical Notes 27, Princeton University Press, Princeton 1981.

[W] A. Weinstein, Periodic orbits for convex hamiltonian systems, Ann. Math. 108 (1978), 507-518.

Massachusetts Institute of Technology

Department of Mathematics

77 Massachusetts Avenue

Cambridge, MA 02139-4307

Email address: xinzhou@math.mit.edu 